\title{
Surprises in the design of anion receptors: calorimetry prevents false reasoning
}

\author{
Vinod D. Jadhav and Franz P. Schmidtchen*
}

Technical University of Munich, Department of Chemistry Lichtenbergstraße 4, D-85747 Garching, Germany

\section{Supporting Information:}

Synthetic outline with some characterization data for the compound $\mathbf{1}$ and its intermediates $(\mathbf{1 1}, \mathbf{1 2})$.<smiles>N#CN(CCI)CCI</smiles>

9

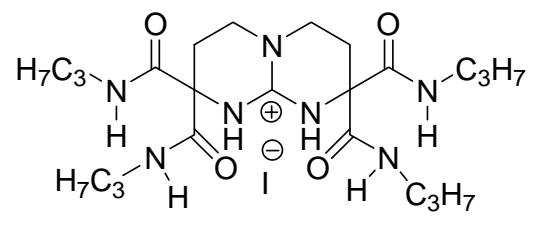

1
$\stackrel{\mathrm{NaH}, \mathrm{DMF}}{\longrightarrow}$<smiles>C1CCCC1</smiles>

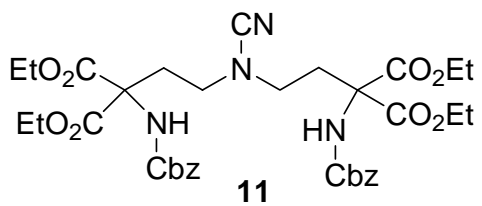

11 i. $\mathrm{HCl}, \mathrm{EtOH}$ ii. $\mathrm{Pd}-\mathrm{C}, \mathrm{H}_{2}$ iii. $\mathrm{Et}_{3} \mathrm{~N}, \mathrm{EtOH}$

$\downarrow$

12

\section{Characterization:}

Guanidinium tetrapropylamide 1. ( as a $\mathrm{Br}^{-}$salt) $\mathrm{C}_{23} \mathrm{H}_{42} \mathrm{Br} \mathrm{N}_{7} \mathrm{O}_{4}$ (MW. 560).

Elemental anal. for 1-I': (\%): Calc: C, 45.47; H, 6.97; N, 16.14

Found: C, 45.47; H, 6.53; N, 15.83

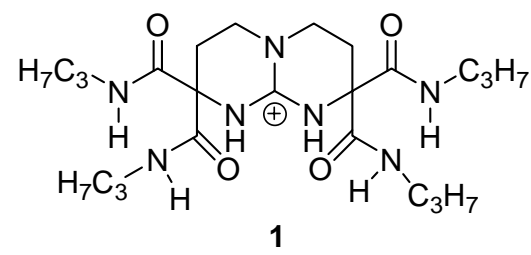

${ }^{1} \mathrm{H}-\mathrm{NMR}$ of 1-Br, $\left(360 \mathrm{MHz} ; \mathrm{CD}_{3} \mathrm{OD}\right): \delta \mathrm{ppm}=8.41$ (bs, guanidinium protons), $8.27(\mathrm{t}, \mathrm{J}=5.7 \mathrm{~Hz}$, amide protons), 3.38 (t, J=5.9 Hz, 4H, N-CH $)_{2}, 3.21$ (t, J= $7.0 \mathrm{~Hz}, 8 \mathrm{H},-\mathrm{NCH}_{2} \mathrm{CH}_{2} \mathrm{CH}_{3}$ ), 2.47 (t, J= 5.90 $\left.\left.\mathrm{Hz}, 4 \mathrm{H},-\mathrm{CH}_{2}\right)^{-}\right), 1.55$ (h, J= 7.2 Hz, 8H, - $\mathrm{NCH}_{2} \mathrm{CH}_{2} \mathrm{CH}_{3}$ ), 0.88 (t, J=7.5 Hz, 12H, $-\mathrm{NCH}_{2} \mathrm{CH}_{2} \mathbf{C H}_{3}$ ).

${ }^{13} \mathrm{C}-\mathrm{NMR}$ of 1-Br, (90.56 MHz; $\mathrm{CD}_{3} \mathrm{OD}$ ): $\delta \mathrm{ppm}=169.17$ (CO, amides), 150.80 (guanidium carbon), 64.88 (quaternary carbons), $45.52\left(\mathrm{~N}-\mathrm{CH}_{2}\right), 43.03\left(-\mathrm{NCH}_{2} \mathrm{CH}_{2} \mathrm{CH}_{3}\right), 28.58$ (aliphatic carbons), 23.47 ($\left.\mathrm{NCH}_{2} \mathrm{CH}_{2} \mathrm{CH}_{3}\right), 11.57\left(-\mathrm{NCH}_{2} \mathrm{CH}_{2} \mathrm{CH}_{3}\right)$. 
Guanidinium tetraethylcarboxilate 12 (as a $\mathrm{Br}^{-}$salt)

$\mathrm{C}_{19} \mathrm{H}_{30} \mathrm{Br} \mathrm{N}_{3} \mathrm{O}_{8}$ (MW 508.38)

Elemental anal. for $12(\%)$ :

Cal: C, 44.89; H, 5.95; N, 8.27

Found: C, 45.03; H, 5.77; N, 8.23.

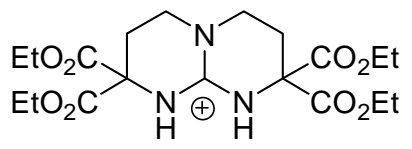

${ }^{1} \mathrm{H}-\mathrm{NMR}\left(360 \mathrm{MHz} ; \mathrm{CD}_{3} \mathrm{CN}\right): \delta \mathrm{ppm}=9.61(\mathrm{~s}, 2 \mathrm{H},-\mathrm{NH}), 4.21$ (q, J= 7.1

12

$\mathrm{Hz}, 8 \mathrm{H},-\mathrm{COOCH}_{2} \mathrm{CH}_{3}$ ), 3.34 (t, J=6.1 Hz, 4H, N-CH $)_{2}, 2.41$ (t, J=6.1 Hz, 4H, -CH $\left.\mathbf{2}^{-}\right), 1.28$ (t, J=7.1 Hz, $\left.12 \mathrm{H},-\mathrm{COOCH}_{2} \mathrm{CH}_{3}\right)$.

${ }^{13} \mathrm{C}-\mathrm{NMR}$ (90.56 MHz; $\left.\mathrm{CD}_{3} \mathrm{CN}\right): \delta \mathrm{ppm}=167.65$ (CO, esters), 151.09 (guanidium carbon), 64.06 ($\left.\mathrm{COOCH}_{2} \mathrm{CH}_{3}\right)$, 63.13(quaternary carbons), $44.37\left(\mathrm{~N}^{\left.-\mathrm{CH}_{2}\right)}, 26.09\right.$ (aliphatic carbons) 14.16 ($\mathrm{COOCH}_{2} \mathrm{CH}_{3}$ ).

Condensation product 11.

$\mathrm{C}_{35} \mathrm{H}_{44} \mathrm{~N}_{2} \mathrm{O}_{12}$ (MW 712.74)

${ }^{1} \mathrm{H}-\mathrm{NMR}\left(360 \mathrm{MHz} ; \mathrm{CDCl}_{3}\right): \delta \mathrm{ppm}=7.31$ (s, 10H, aromatic), 6.15<smiles>CCOCC(CCN(C#N)CCC(C(=O)OCC)(C(=O)OCC)C(=O)OCC)(COCC)NC(=O)OCC</smiles>
(s, 2H, -NH), 5.06 (s, 4H, PhCH $\left.\mathrm{H}_{2} \mathrm{O}\right), 4.20\left(\mathrm{~m}, 8 \mathrm{H},-\mathrm{COOCH}_{2} \mathrm{CH}_{3}\right)$, 2.89 (t, J= $7.1 \mathrm{~Hz}, 4 \mathrm{H}, \mathrm{N}-\mathrm{CH}_{2}$ ), 2.61 (t, J= $7.1 \mathrm{~Hz}, 4 \mathrm{H}$, -aliphatic carbons), 1.20 (t, J= 7.1 Hz, 12H, $\mathrm{COOCH}_{2} \mathrm{CH}_{3}$ ).

${ }^{13}$ C-NMR (90.56 MHz; CDCl3): $\delta=167.23$ (CO, esters), 154.49 (-NCO-), 135.87, 128.49 128.25, 128.17 (aromatic carbons), $116.19(\mathrm{CN}), 67.17$ (benzyl carbons), 64.73 (quaternary carbons), 63.00 ($\left.\mathrm{COOCH}_{2} \mathrm{CH}_{3}\right), 47.42\left(\mathrm{~N}-\mathrm{CH}_{2}\right), 30.84$ (aliphatic carbons) $13.79\left(-\mathrm{COOCH}_{2} \mathrm{CH}_{3}\right)$.

Table 1: The binding energetics of 1:1 stoichiometric complexes of guest species 3-8 (as a TBA salt) to guanidinium hosts $\mathbf{1}$ and $\mathbf{2}$ in acetonitrile (supporting material for figure $\mathbf{4}$ )*.

\begin{tabular}{|l|c|c|c|c|c|c|c|c|c|c|c|c|}
\hline Guest & \multicolumn{2}{|c|}{$\mathbf{3}$} & \multicolumn{2}{|c|}{$\mathbf{4}$} & \multicolumn{2}{|c|}{$\mathbf{5}$} & \multicolumn{2}{|c|}{$\mathbf{6}$} & \multicolumn{2}{|c|}{$\mathbf{7}$} & \multicolumn{2}{|c|}{$\mathbf{8}$} \\
\hline Host & $\mathbf{1}$ & $\mathbf{2}$ & $\mathbf{1}$ & $\mathbf{2}$ & $\mathbf{1}$ & $\mathbf{2}$ & $\mathbf{1}$ & $\mathbf{2}$ & $\mathbf{1}$ & $\mathbf{2}$ & $\mathbf{1}$ & $\mathbf{2}$ \\
\hline $\mathbf{T} \Delta \mathbf{S}$ & 3.15 & 1.40 & 4.31 & 0.26 & 4.02 & 2.39 & 2.40 & 1.36 & 3.02 & -0.10 & 7.22 & 3.46 \\
$-\Delta \mathbf{H}$ & 4.21 & 5.32 & 4.19 & 5.59 & 2.46 & 3.50 & 4.92 & 5.13 & 5.40 & 7.93 & 2.80 & 2.84 \\
$-\Delta \mathbf{G}$ & 7.36 & 6.73 & 8.50 & 5.84 & 6.49 & 5.90 & 7.32 & 6.50 & 8.42 & 7.84 & 10.00 & 6.32 \\
\end{tabular}

* All values are in $\mathrm{kcal} \mathrm{mol}^{-1}$. 